\title{
VOCO Dental Challenge 2015: Herausforderung für junge Forscher
}



Am 25. September 2015 findet zum 13. Mal die VOCO Dental Challenge in Cuxhaven statt. Im modernen Schulungszentrum haben junge Wissenschaftler die einmalige Gelegenheit, ihre Forschungsarbeiten innerhalb eines exklusiven Fachkreises zu präsentieren und Erfahrungen in der Diskussion zu sammeln. Erfahrungen, die seit der Premiere des renommierten Wettbewerbs im Jahr 2003 den beruflichen Werdegang vieler Teilnehmer nachhaltig positiv beeinflusst haben. Präsentationstraining, Reputationserhöhung und das Knüpfen wertvoller Kontakte sind dabei nur einige Vorzüge der VOCO Dental Challenge. Neben der fachlichen Herausforderung und den attraktiven Geldpreisen lockt zudem ein interessantes Programm, das Wissenschaft und Unterhaltung verbindet.

Teilnehmen können Studenten sowie Absolventen, die ihr Studium 2010 oder später beendet haben. Eine weitere Teilnahmevoraussetzung ist die Präsentation einer Studie, an der zumindest ein VOCOPräparat beteiligt ist. Außerdem dürfen die Untersuchungsergebnisse vor dem 30.04.2015 noch nicht öffentlich präsentiert worden sein. Die Anmeldung und Abgabe der einzureichenden Unterlagen (Abstract von ca. einer A4-Seite, Lebenslauf) hat spätestens bis zum 30.04.2015 zu erfolgen (weitere Informationen hierzu auch auf voco.de).

Nach einer Pressemitteilung der

voco GmbH, Cuxhaven 